\title{
Control de la gestión del destino turístico sucre- san vicente - jama - pedernales, caso observatorio turístico
}

\author{
DOI: $10.46932 / \operatorname{sjd} d v 2 n 2-104$
}

Received in: March 1st, 2021

Accepted in: May 30th, 2021

\section{Gema Viviana Carvajal Zambrano}

Magíster en Administración de Empresas con mención en Marketing, Ingeniera Comercial mención proyectos de inversión

Institución actual: Universidad Laica Eloy Alfaro de Manabí extensión Bahía de Caráquez, Manabí

Dirección completa: Avenida Cesar Rupertí y calle Antonio Oramas, Leonidas Plaza Bahía de

Caráquez, cantón Sucre, provincia Manabí. Ecuador.

Correo electrónico:vivicarvajalzambrano@gmail.com

\section{Ericka Vanessa Almeida Lino}

Magíster en Telecomunicaciones, Ingeniera en Sistemas Informáticos.

Institución actual: Universidad Laica Eloy Alfaro de Manabí extensión Bahía de Caráquez, Manabí.

Dirección completa: Avenida Cesar Rupertí y calle Antonio Oramas, Leonidas Plaza Bahía de Caráquez, cantón Sucre, provincia Manabí. Ecuador.

\section{Frank Ángel Lemoine Quintero}

Doctorado en Ciencias Económicas, Master MBA.

Institución actual: Universidad Laica Eloy Alfaro de Manabí extensión Bahía de Caráquez, Manabí.

Dirección completa: Avenida Cesar Rupertí y calle Antonio Oramas, Leonidas Plaza Bahía de Caráquez, cantón Sucre, provincia Manabí. Ecuador.

\section{Eduardo Antonio Caicedo Coello}

Doctor en ciencias Pedagógicas, Licenciado en ciencias dela educación . Espec. Física y matemáticas. Institución actual: Universidad Laica Eloy Alfaro de Manabí extensión Bahía de Caráquez, Manabí.

Dirección completa: Avenida Cesar Rupertí y calle Antonio Oramas, Leonidas Plaza Bahía de Caráquez, cantón Sucre, provincia Manabí. Ecuador.

Correo electrónico: eduardocaicedoec@gmail.com

\section{Luis Daniel Zambrano Molina}

Magister en exploración, explotación y producción de petróleo. Ingeniero mecánico.

Institución actual: Universidad Laica Eloy Alfaro de Manabí extensión Bahía de Caráquez, Manabí.

Dirección completa: Avenida Cesar Rupertí y calle Antonio Oramas, Leonidas Plaza Bahía de Caráquez, cantón Sucre, provincia Manabí. Ecuador.

Correo electrónico: danielzambrano10@hotmail.com

\section{Carlos Subía Veloz \\ Ingeniero comercial, CPA.}

Institución actual: Universidad Laica Eloy Alfaro de Manabí extensión Bahía de Caráquez, Manabí.

Dirección completa: Avenida Cesar Rupertí y calle Antonio Oramas, Leonidas Plaza Bahía de Caráquez, cantón Sucre, provincia Manabí. Ecuador.

Correo electrónico: robertosubiaveloz@hotmail.com 


\section{Jennifer Doris Pisco García}

Economista, Ingeniera en Contabilidad y Auditoría

Universidad Laica Eloy Alfaro de Manabí extensión Bahía de Caráquez, Manabí.

Avenida Cesar Rupertí y calle Antonio Oramas, Leonidas Plaza Bahía de Caráquez, cantón Sucre, provincia Manabí. Ecuador.

Correo electrónico: jpisco_10584@ hotmail.com

\section{Dianelys Nogueira Rivera}

PhD Technical Sciences, Magíster en Gestión de la Producción, Ingeniera Industrial. Institución actual: Universidad de Matanzas Facultad de Ciencias Empresariales (Cuba).

Dirección completa: Matanzas, Cuba, Correo electrónico: nelydaylinyuly@ gmail.com

\section{RESUMEN}

La presente investigación se realiza en el destino turístico costa norte y tiene como objetivo la creación de un Observatorio Turístico que permitirá: analizar la información turística relevante, que facilite la toma de decisiones. El desarrollo del observatorio se realizará por etapas: la primera consiste en la actualización y diagnóstico de la situación actual de atractivos, instalaciones e infraestructura turística. Una segunda etapa que prevé la estructuración de un sistema de indicadores de gestión turística y de información que facilite la toma de decisiones, a través de un cuadro de mando integral. Otra etapa que consiste en la realización de inteligencia de mercados y una última etapa en la que se pretende brindar servicios informáticos a empresas turísticas, no turísticas y de agencia de viajes virtual. Entre las principales herramientas utilizadas se encuentran la revisión documental, el análisis de indicadores, los métodos de experto Delphi y Kendall, encuestas, entrevistas, análisis de las cinco fuerzas de la competencia, entre las fundamentales. Para el procesamiento y análisis de información Excel, SPSS, Access. Como principales resultados de la investigación se puede mencionar: la creación del observatorio turístico para el registro, análisis y procesamiento de la información que facilite la toma de decisiones en el destino Sucre-San Vicente- Jama - Pedernales, la actualización y diagnóstico de la situación real del destino, la creación y gestión de un sistema de indicadores que resumidos en un cuadro de mando integral, desarrollar la inteligencia de mercado y brindar servicios informáticos a las empresas que lo requieran.

Palabras claves: Observatorio turístico, indicadores, control de gestión, efectividad, toma de decisiones.

\section{INTRODUCCIÓN}

El desarrollo del turismo en el Ecuador tiene el reto de convertir el país en un destino de referencia y contribuir decisivamente al cambio de la matriz productiva, convirtiéndose en el primer sector de ingreso no petrolero.

Dentro de los cuatro mundos existentes para el desarrollo sostenible se encuentra Galápagos, la Amazonía, La Sierra y el destino Costa; todos con amplia diversidad y con un conjunto de atractivos potenciales que pueden ser explotados sostenidamente, ofreciendo beneficios sustanciales a la economía del país. En el mundo de la Costa y específicamente en el destino costa norte existe un número de atractivos que poseen vocación y tradición turística importante, los cuales permiten desarrollar las 11 
líneas de productos establecidas por el Ministerio de Turismo (MINTUR) en el Plan Integral de Marketing Turístico del Ecuador (PIMTE):

1. Circuitos turísticos generales

2. Sol y playa

3. Turismo comunitario

4. Turismo cultural: mercados y artesanías

5. Ecoturismo y turismo de naturaleza: observación de flora y fauna (observación de ballenas)

6. Parque temático

7. Deportes y Aventura: Surf

8. Agroturismo

9. Turismo de salud

10. MICE

11. Cruceros

Pese a los atractivos y potencialidades turísticas que posee Ecuador y al crecimiento que ha experimentado la actividad turística en los últimos años aun el país no logra consolidarse como un destino turístico en el área Sudamérica, ocupando el séptimo lugar en el posicionamiento por países del arrea; superado por Argentina, Brasil, Perú, Uruguay, Colombia y Chile. Por otra parte, el crecimiento del turismo se ha centrado en productos estrellas o mundo como Galápagos, Quito y Cuenca; mientras que el mundo de la costa no ha alcanzado un crecimiento significativo en lo que respecta al turismo internacional. Esta situación se agudiza por el terremoto del 16A que afecto sensiblemente la estructura turística del destino costa.

El destino turístico proyectado abarca los cantones Sucre- San Vicente- Jama- Pedernales los cuales no escapan de las dificultades anteriores y que además de su gran afectación enfrentan el riesgo del temor del turista a visitar la zona. No obstante, es tradicional en estos territorios desarrollar una actividad turística desarticulada, carente de un enfoque integral y muy orientado al turismo nacional, de bajos ingresos y pocos exigentes.

Es característico del destino poseer una marcada estacionalidad, que se concentra en arribos en feriados, un bajo porcentaje de ocupación que no excede el $15 \%$ lineal en el año, bajo niveles de calidad de servicio y una pobre gestión ambiental.

La necesidad de una gestión integral o de destino turístico que garantice la sostenibilidad y la efectividad del sistema turístico es vital para el mundo de la costa y el destino objeto de estudio. Por lo 
que se define como problema general de la investigación la carencia de un sistema de control de gestión que facilite la toma de decisiones efectivas en el destino turístico Sucre- San Vicente- Jama- Pedernales.

Otros de los documentos rectores del desarrollo turístico del Ecuador es el Plan Nacional de Competitividad turística, que establece 12 factores claves de los cuales se considera que este proyecto incide en los que a continuación se detallan:

1. El factor de éxito es impactado directamente por el proyecto brindando información, oportuna, veraz y en tiempo real, que permita establecer un sistema informativo y de indicadores que facilite el control de gestión de destino y la toma de decisión; a través de un observatorio turístico y con un cuadro de mando integral. Que aporta directamente con el

1. Desarrollo tecnológico, formación y capacitación profesional

2. Ambiente hospitalario, cultura de servicio, facilitación y seguridad: el proyecto contribuirá a este factor clave con dos de las perspectivas del cuadro de mando integral, perspectiva ambiental con indicadores de calidad de la playa, playa con certificación bandera azul y otros indicadores ambientales; la hospitalidad y cultura de servicio será impactada con las perspectivas de procesos internos y clientes especialmente con la satisfacción de los clientes, número de quejas y reclamos e imagen entre otros.

3. Marco jurídico y normatividad: el proyecto contribuirá en la parte normativa al establecer un sistema de información e indicadores general para el destino que establecerá el control de gestión facilitando la toma de decisiones; y en marco jurídico solo si el MINTUR lo establece.

4. Apoyo gubernamental a la inversión y cargas fiscales al sector y al turista, Que la perspectiva financiera es la que va a tributar, más eficiencia es igual a más inversión

5. Mercadeo y comercialización, el proyecto facilitará la inteligencia del mercado en la perspectiva cliente, va a ser inteligencia de mercado segmentado por imagen, posicionamiento y estudio de necesidades de cliente, dentro de mercadeo promoción, seguimiento de los mercados metas, mercados consolidación y mercado de oportunidades.

6. Gestión de calidad, esa es impactada directamente por la perspectiva del cliente, a través del análisis de la satisfacción de los clientes internos y externos y la imagen.

7. Medición de la productividad, por la perspectiva procesos internos y formación. La preparación del personal contribuye directamente a la productividad del personal y el incremento de la eficacia de los procesos internos también impactan a la productividad al existir menos reprocesos, re trabajo, quejas y reclamaciones. 
8. Orientación empresarial y del sector público hacia el mercado externo, que, aunque el sistema de indicadores y observatorio gestionará el destino, aunque el proyecto se monitoree se va a ser énfasis en la inteligencia del mercado internacional.

9. Articulación de la cadena de valor, el sistema informativo que se implantará establecerá indicadores para toda la cadena de valor tanto para las empresas turísticas y no turísticas lo que contribuye a su integración.

10. Rivalidad competitiva y vocería del sector, en la perspectiva cliente se harán estudio de imagen, posicionamiento de la competencia.

El proyecto se elaborará en total correspondencia con los objetivos establecidos en el PLANDETUR 2020, que a continuación se detallan:

- Generar un proceso orientador que coordine los esfuerzos públicos, privados y comunitarios para el desarrollo del turismo sostenible, basado en sus territorios y bajo los principios de alivio a la pobreza, equidad, sostenibilidad, competitividad y gestión descentralizada.

- Crear las condiciones para que el turismo sostenible sea un eje dinamizador de la economía ecuatoriana que busca mejorar la calidad de vida de su población y la satisfacción de la demanda turística actual, aprovechando sus ventajas comparativas y elementos de unicidad del país.

- Insertar al turismo sostenible en la política de Estado y en la planificación nacional para potenciar el desarrollo integral y la racionalización de la inversión pública y privada.

En estos objetivos las cuatro perspectivas se encuentran involucradas debido a que PLANDETUR desea mejorar a través del turismo sostenible la calidad de vida de las poblaciones, por medio de la realización de proyectos que favorezcan a las comunidades incrementando sus ingresos, lo cual se podría realizar asertivamente si se posee información que permita medir la factibilidad y sostenibilidad de los mismos.

Se puede referenciar como ejemplo de todo este destino a el cantón Sucre que tiene como cabecera cantonal a la ciudad de Bahía de Caráquez, su nombre se debe a la Bahía formada por la desembocadura del río Chone y a los primeros pobladores que fueron los Caras. Bahía de Caráquez cuenta con un gran atractivo turístico y frente a ella está San Vicente, cruzando en lancha, gabarra o por vía terrestre y cuenta con el aeropuerto "Los Perales". De norte a sur existen amplias playas islas e islotes, lugares que son preferidos por los turistas. Se pueden observar manglares y aves marinas. Río Muchacho es una buena elección para el turismo ecológico y de aventura. (Lemoine Quintero, Hernández Rodríguez, Castro Robles, \& Zambrano Arteaga, 2021) 


\section{MARCO TEÓRICO}

En la escasa literatura del tema no existen definiciones concretas acerca de que es un observatorio turístico. Este hecho puede deberse a que la palabra "observatorio", ya que por sí misma nos está indicando la focalización de uno o varios temas o sector específico.

En este sentido, el Dr. Enrique Torres Bernier afirma que: “el observatorio turístico es, ante todo, un informe de coyuntura que, como todos, nos proporciona información sobre un campo concreto de la actividad humana, el turismo, que ayuda a la toma de decisiones y al desarrollo del planeamiento."

De un modo más concreto, agrega, "se puede decir que un observatorio turístico es:

Un instrumento que ayuda a la toma de decisiones de la iniciativa privada (empresarios) y pública (administraciones) de un territorio que se identifica como un destino turístico, real o potencial.

Dentro del Cuadro de Mando Integral (herramienta de gestión) y sus perspectivas es donde se realizará el seguimiento a través de las cuatro que este posee, como son: Clientes, Financiera, Formación y Procesos Internos; pero que para este caso se han unificado dos perspectivas (Formación y Procesos Internos) y se agrega la perspectiva ambiental.

Perspectiva de Clientes es donde se estará midiendo la calidad de servicio, perspectiva del proceso, se tendría en cuenta cómo manejar la satisfacción del cliente y mejorar la calidad.

Perspectiva Financiera o Económica con unos buenos indicadores que faciliten el control de gestión la eficiencia tiende a maximizar beneficio o a propiciar la inversión en el sector del turismo, que mejoraría la calidad. Mediante dos dimensiones la técnica y la asignativa. La eficiencia asignativa depende de la eficiencia técnica. Técnica es la base de la eficiencia asignativa (promedio de trabajadores, productividad de trabajadores).

Perspectiva de Formación se medirá a través de promedios de trabajadores y porciento de trabajadores preparados en la función de turismo es decir que si están capacitados. Eficiencia: Costos de Calidad, formación, y costos de no calidad (son costos de fallo) o mal proceso de comercialización, costo de atender quejas, \% de habitaciones vacías. Turistas que se deja de recibir por mala gestión. Costos de reclamos Perspectiva de Procesos internos se van a seguir con la satisfacción de los clientes, indicadores de personal.

Perspectiva de Ambiental es donde se va a considerar aspectos como calidad o certificación de playas e impactos ambiental en general.

Muy relacionado con el diseño de Sistemas de Control de Gestión se encuentra también el tema del Cuadro de Mando Integral -Balanced Scorecard- donde se considera que el mismo proporciona a los ejecutivos un amplio marco que traduce la visión y estrategia de una empresa en un conjunto coherente de indicadores de actuación. 
Muchas personas piensan que los indicadores son una herramienta para controlar el comportamiento y para evaluar la actuación pasada. No debemos verlos solo así, sino incorporados al Cuadro de Mando Integral (CMI) para articular y comunicar la estrategia empresarial, la estrategia del negocio y para coordinar y alinear las iniciativas individuales de la organización y la de sus múltiples departamentos a fin de conseguir un objetivo común. El CMI no se esfuerza por hacer que los individuos y las unidades de la Organización sigan un plan preestablecido, el objetivo tradicional de un sistema de control. El CMI debe ser utilizado como un sistema de comunicación, de información y de formación, y no como un sistema de control.

El cumplimiento de los cuatro pilares del CMI contribuye, en mucho: a la motivación de los empleados; a mejorar todas las etapas de la Cadena de Valor; a satisfacer las expectativas de los clientes y conseguir su lealtad; por último, a ofrecer mayores rendimientos económicos a los accionistas. (Campaña, 2003).

En términos generales, se puede decir que el control debe servir de guía para alcanzar eficazmente los objetivos planteados con el mejor uso de los recursos disponibles (técnicos, humanos, financieros, etc.). Por ello podemos definir el control de gestión como un proceso de retroalimentación de información de uso eficiente de los recursos disponibles de una empresa para lograr los objetivos planteados.

Se entiende por control de gestión el conjunto de procesos que la empresa aplica para asegurarse de que las tareas que en la misma se realizan están encaminadas a la consecución de sus objetivos.

Para Pérez Campaña (2003) el Control de Gestión es una de las herramientas fundamentales para que las empresas puedan obtener productos y servicios con una eficiencia relevante, que le permitan el acceso al mundo competitivo de hoy, se ha convertido en un tema de gran actualidad.

Un sistema informático es un sistema de información que está informatizado. No todos los sistemas de información son sistemas informáticos, pero todos los sistemas informáticos son sistemas de información. Por lo tanto, se puede decir que un sistema informático es un subconjunto de un sistema de información.

Los indicadores de calidad son instrumentos de medición, de carácter tangible y cuantificable, que permiten evaluar la calidad de los procesos, productos y servicios para asegurar la satisfacción de los clientes. Dicho de otro modo, miden el nivel de cumplimiento de las especificaciones establecidas para una determinada actividad o proceso empresarial.

Los indicadores de gestión miden, de manera global, el resultado final de las actividades empresariales basándose en un estándar, el cual responde al nivel de calidad objetivo que la empresa espera y desea alcanzar. 
La gestión por procesos busca reducir la variabilidad innecesaria que aparece habitualmente cuando se producen o prestan determinados servicios y trata de eliminar las ineficiencias asociadas a la repetitividad de las acciones o actividades, al consumo inapropiado de recursos, etc. Facilita la coordinación y la sinergia entre los diferentes procesos que se dan en una organización y es una base sólida para organizar el empowerment, la asunción de responsabilidad por parte de los profesionales en el ámbito de actuación de su proceso y su trabajo diario. (Rubio, 2006)

\section{MATERIAL Y MÉTODO}

La investigación utiliza el método descriptivo y utiliza la investigación cuantitativa y cualitativa, entre las principales herramientas utilizadas se pueden citar: la revisión de fuentes bibliográficas, la aplicación del método de experto Delphi y Kendall, la observación, la entrevista, la encuesta y análisis de indicadores. Los temas tratados explican la adecuada utilidad que van a dar los expertos basándose en los criterios de turismo y gestión.

\section{RESULTADOS}

- Un modelo para el control de la gestión del destino Sucre-San Vicente- Jama - Pedernales, basado en la integración de un observatorio turístico y un cuadro de mando integral para facilitar la gestión de la eficacia, la eficiencia, la competitividad y el proceso de toma de decisiones en el destino.

- El modelo y los procedimientos están en correspondencia con las tendencias actuales analizadas en el estado del arte y de la práctica.

- Un procedimiento específico de diagnóstico de las dificultades que se presenta el control de gestión en el destino Sucre- San Vicente- Jama - Pedernales.

- Creación de un procedimiento que norma el trabajo del observatorio turístico del destino.

- Elaborar el cuadro de mando integral que facilitaría el control de gestión y el proceso de la toma de decisiones en el destino.

- La creación de un sistema de indicadores para el control de la gestión de todas las empresas que componen el destino.

\section{CONCLUSIÓN}

El desarrollo de este proyecto contribuirá a la obtención de la sostenibilidad y competitividad turística enfocado en la zona norte de Manabí, este se sumaría al observatorio de Galápagos, y el de 
Guayaquil siendo el tercero que se implementaría en Ecuador, debido a que las demás ciudades se basan en estadísticas emitidas por el Ministerio de Turismo y otras entidades.

La investigación que resulten del observatorio permitirá posteriormente poder contar con indicadores que reflejen datos reales y actuales que nos permitan analizar la situación actual de la actividad turística, en base a las cuatro perspectivas cliente, formación y procesos internos, ambiental y financiera que permitirán contribuir con el PLANDETUR, Plan Nacional de Desarrollo, Plan Nacional de Competitividad Turística y MINTUR del Ecuador.

La información recabada permitirá determinar la factibilidad y sustentabilidad en la implementación de futuros proyectos turísticos; así mismo mejorar los procesos orientadores que permitan crear las condiciones para que el turismo sostenible sea un eje dinamizador de la economía ecuatoriana. 


\section{REFERENCIA BIBLIOGRÁFICA}

Batista, J. (26 de 04 de 2011). Buenas Tareas. Recuperado el 23 de 09 de 2015, de http://www.buenastareas.com/ensayos/Introduci\%C3\%B3n-Al-Control-De-Gesti\%C3\%B3nDe/2010684.html

Bou, R. G. (S/D de S/M de S/A). Gestion . Org. Recuperado el 23 de 09 de 2015, de http://www.gestion.org/estrategia-empresarial/4594/que-es-el-control-de-gestion/

Campaña, M. P. (14 de 09 de 2003). El sistema de control de gestión. Conceptos básicos para su diseño. Recuperado el 21 de 09 de 2015, de http://www.gestiopolis.com/sistema-control-gestion-conceptosbasicos-diseno/

DE, D. (2008-2015). (G. c. WordPress, Editor) Recuperado el 18 de 09 de 2015, de Definición. DE: http://definicion.de/gestion/

GAIBOR., L. H. (2011). Recuperado el 21 de 09 de 2015, de http://dspace.utpl.edu.ec/bitstream/123456789/2039/3/Benavides_Gaibor_Luis_Hernan.pdf

Ing. Industrial-administrador, a. E., productividad, c. y., \& educación. (S/F). Herramientas para sistemas de Calidad ISO 9001- Normas 9000.com. (Origen: Artículos gratuitos de ArticuloZ.com ) Recuperado el 21 de 09 de 2015, de http://www.normas9000.com/importancia-gestion-calidad.html

Lemoine Quintero, F., Hernández Rodríguez, N., Castro Robles, J., \& Zambrano Arteaga, K. (Abril de 2021). South Florida Journal of Development. doi:10.46932/sfjdv2n

MX, D. (S/F). Definición. MX Miles de términos explicados. Recuperado el 18 de 09 de 2015, de http://definicion.mx/gestion/

Render, H. y. (2009). Principios de Administración de operaciones. Recuperado el 18 de 09 de 2015, de http://www.eumed.net/libros-gratis/2013a/1321/gestion.html

Rubio, J. A.-J.-S. (2006). Manuales de Dirección Médica y Gestión Clínica. (L. Á. Ochoa, Ed.) Ediciones Díaz de Santos . Recuperado el 21 de 09 de 2015, de https://books.google.com.ec/books?id=bVbxvdyVjM8C\&pg=PA304\&lpg=PA304\&dq=Gesti\%C3\%B3 n-

Conceptos,+importancia $+y++$ beneficio\&source $=$ bl\&ots=CEcdfHWHJr\&sig=xh_2Ry5TNZDgei0X51v DfS_7A0s\&hl=es-419\&sa=X\&ved=0CCgQ6AEwAmoVChMI-aC4K6IyAIVyCceCh2msgIv\#v=onepage \&q=Gest

Santágata, M. H. (11 de 2010). La importancia de un observatorio turístico en provincia de buenos aires como instrumento de orientación en la toma de decisiones y en la planificación. Recuperado el 24 de 09 
de

2015 ,

de

http://sedici.unlp.edu.ar/bitstream/handle/10915/25780/Documento_completo.pdf?sequence=1

Significados"Gestión". (S/F). Significados"Gestión". Recuperado el 18 de 09 de 2015, de http://www.significados.com/gestion/

Turismo, M. d. (18 de 11 de 2013). turismo.gob.ec. Recuperado el 23 de 09 de 2015, de http://www.turismo.gob.ec/wp-content/uploads/2015/04/Estatuto-Org\%C3\%A1nico-por-Procesos.pdf

Turística, S. d. (S/D de S/M de S/A). ISQ turística Implantación de Sistemas de Qualitat S.L.N.E. Recuperado el 23 de 09 de 2015, de http://www.isq-turistica.com/sectores-tur\%C3\%ADsticos/

Velastegui, W. (06 de 08 de 2013). slideshare. Recuperado el 23 de 09 de 2015, de http://es.slideshare.net/wilsonvelas/control-de-gestion-24989890 